\title{
El efecto de la estructura de la escuela primaria en el funcionamiento de Comunidades Profesionales de Aprendizaje (CPAs): Un estudio de caso múltiple
}

\author{
Eduardo Flores-Kastanis \\ Facultad de Filosofía y Letras \\ Universidad Autónoma de Chihuahua \\ efloresk@uach.mx
}

\begin{abstract}
Resumen
El estudio busca identificar posibles efectos de la estructura de la escuela primaria sobre el funcionamiento de las Comunidades Profesionales de Aprendizaje (CAPs). Ser presentan los resultados parciales de una investigación terminada en lo que se refiere a las 3 escuelas de la ciudad de Chihuahua. En estas tres escuelas se realizó una intervención para facilitar la creación y funcionamiento de CPAs, pidiendo a los maestros que libremente seleccionaran una programa estratégico de la escuela que les interesara resolver, y dándoles tiempo y márgenes de decisión amplios para poder establecer y trabajar en su agenda dentro de su margen de trabajo, Se realizó un estudio de casos múltiple con las 3 escuelas, buscando los patrones similares a las 3. Se encontró que la práctica común de crear "comisiones" parece tener un efecto debilitador en las CPAs. Dos CPAs lograron mejoras en su práctica, en el entendimiento de esa práctica, y en las condiciones bajo las cuáles la práctica se lleva a cabo cuando no afectó (ni para bien ni para mal) la práctica de "asignar comisiones" en la composición del grupo o en la estrategia sobre la cuál querían trabajar. En general 7 de 10 CPAs lograron mejoras en sus escuelas, aunque cinco de las CPAs no lograron cambios en su práctica individual ni mejoraron las condiciones organizacionales bajo las cuales trabajan.
\end{abstract}

\section{Palabras clave}

Comunidades Profesionales de Aprendizaje, Educación Básica, Aprendizaje organizacional, Organización Escolar, Trabajo docente.

\section{Introducción}

Presentamos resultados parciales de un proyecto de investigación realizado con escuelas primarias públicas en Chihuahua, Monterrey y Hermosillo donde se llevó a cabo una intervención para facilitar la formación de comunidades profesionales de aprendizaje (CPAs) relacionadas con proyectos de mejora consensuadas por los maestros y directores. El proyecto busca responder la pregunta: ¿Cómo afecta la estructura funcionamiento de Comunidades Profesionales de Aprendizaje (CPAs) en la escuela primaria? y a la vez contribuir a resolver problemas prácticos presentes en escuelas primarias públicas en México, para aumentar la probabilidad de que los mismos maestros mejoren su trabajo y las condiciones bajo las cuáles ese trabajo se realiza.

\section{Planteamiento del Problema}

En los últimos años se ha generalizado la 
idea en el sistema educativo público sobre las ventajas de establecer CPAs entre maestros de escuelas de educación básica. Las CPAs son grupos de maestros que se integran entre colegas de la misma escuela o de otras escuelas al compartir un interés común establecido por ellos mismos, con el propósito de contar con un espacio de interacción regular que les permita aprender más sobre su tema de interés, y realizar de manera conjunta acciones planteadas por ellos mismos que mejoren los resultados de su trabajo en formas que sean aceptables para sus integrantes y sostenibles a largo plazo (DuFour, Eaker, \& DuFour, 2005; Lieberman \& Miller, 2008; McLaughlin \& Talbert, 2001, 2006; Whitford \& Wood, 2010). Esta práctica, como otras que se han planteado en la literatura sobre administración educativa en los últimos 10 años, se deriva de la investigación cada vez más frecuente sobre aprendizaje organizacional en escuelas en muchos países (Stoll et al, 2006), incluyendo México (Flores Kastanis, et al., 2007, 2009.

La tendencia a implantar este tipo de práctica, se justifica en términos de supuestas ventajas asociadas a esta forma de trabajo y como una respuesta a los problemas del sistema educativo. Desde una perspectiva eminentemente racional tienen sentido y difícilmente podrían refutarse. Sin embargo, y a pesar del atractivo racional que despierta la idea de las CPAs, hay muy poca evidencia empírica de lo que realmente ocurre cuando se pone en práctica esta forma de trabajo en las escuelas. Esta ausencia de evidencia empírica es importante ya que la investigación sobre aprendizaje organizacional parte del reconocimiento de que la actual estructura organizacional de la escuela, derivada de principios eminentemente industriales, actúa en contra del trabajo de conocimiento que caracteriza mejor el trabajo del maestro que el trabajo industrial (Flores-Kastanis, 2010). Las CPAs, como práctica, buscan ser una alternativa a la organización industrial que fundamenta la estructura de la escuela. Sin embargo se intentan implantar en escuelas que mantienen esa estructura tradicional. Implantar la práctica de las CPAs requiere, en consecuencia, ajustes en la estructura. Pero qué tanto necesita cambiar la estructura para facilitar el trabajo de conocimiento sigue siendo, en muchos sentidos, un planteamiento meramente teórico. Sin investigación empírica que valide estos planteamientos teóricos, los cambios en las prácticas administrativas de la escuela tendrán un efecto limitado, e incluso contraproducente. Ejemplos de esta problemática abundan en las iniciativas actuales que pretenden innovar la gestión en las escuelas de nivel básico sin hacer ningún cambio en las condiciones organizacionales bajo las cuáles estas nuevas formas de gestión deben llevarse a cabo. No sólo no mejoran la situación. Incluso empeoran la persistente sensación de sobrecarga que los maestros vienen sufriendo desde mediados de la década pasada (Hargreaves, 1996).

Ya existe investigación en primarias mexicanas que ha ayudado a identificar algunos efectos de la estructura organizacional sobre el trabajo del maestro (ver a Flores-Kastanis et al 2007, Flores-Kastanis et al 2009, Flores Fahara y Torres Herrera, 2010, Flores-Kastanis y Ruiz Cantisani, en prensa). Entre otros se ha encontrado que el aprendizaje organizacional se ve favorecido en las escuelas por las disciplinas del dominio personal y el uso de diferentes modelos mentales, mientras la ausencia de 
pensamiento sistémico y la dificultad de generar una visión compartida generada por la estructura lo dificulta, siguiendo el modelo de Senge (2006) sobre aprendizaje organizacional. La generación de soluciones independientes es el activo de conocimiento más importante con el que cuentan las escuelas y que se favorece y protege con la estructura actual de la escuela (Flores-Kastanis et al 2007). Pero el compartir abiertamente estas soluciones novedosas se ve coartado por una cultura escolar que castiga el compartir formas diferentes de trabajar en la escuela (Flores-Kastanis et al 2009), aunado a la importancia excesiva que le da el maestro a "su grupo" y a "cumplir con el programa” (Flores-Kastanis y Ruiz Cantisani, en prensa). Esta cultura reduce considerablemente el valor de generar soluciones independientes. El conocimiento generado con estas soluciones se comparte poco o no se comparte, reduciendo enormemente la posibilidad de que sea reutilizado por otros maestros.

Este conocimiento sobre los efectos de la estructura sobre el trabajo del maestro permitió identificar algunos aspectos estructurales que podrían modificarse para facilitar, por lo menos teóricamente, el funcionamiento de CPAs en las escuelas públicas. Sin embargo, los efectos derivados de cambios estructurales necesitan validarse empíricamente. En función de este propósito, la pregunta de investigación de este proyecto es:

¿Cómo afecta la estructura organizacional el funcionamiento de Comunidades Profesionales de Aprendizaje (CPAs) en la escuela primaria?

\section{Método}

Se realizó un estudio de casos múltiple
(Stake, 2006; Yin, 2009) de octubre de 2011 a junio de 2012 con la ayuda de una intervención utilizada cómo "disparador". La investigación no busca evaluar la intervención. La intervención se lleva a cabo para generar un evento que permita responder la pregunta de investigación, ya que en condiciones normales la situación de interés no se presenta. El uso de intervenciones para generar condiciones que no se presentan de manera cotidiana no es nuevo, sobre todo en el caso de prácticas altamente institucionalizadas como la organización escolar. La investigación-acción parte de este supuesto. Durante más de 30 años y en diferentes disciplinas se ha realizado este tipo de investigación (ver a (Noffke \& Somekh, 2009) para educación, y a Reason \& Bradbury (2008) para otras disciplinas). El uso de "disparadores" combinado con otros métodos, como el estudio de casos múltiple (Yin, 2009; Stake, 2006) es relativamente nuevo, pero ha probado ser efectivo para entender mejor innovaciones que buscan cambiar condiciones existentes más que prácticas específicas. Permite plantear, como argumenta Yin (2009), generalizaciones analíticas. Si en $\mathrm{X}$ número de casos independientes se presentan las mismas características podemos suponer que también se presentarán en otros, mientras no haya evidencia en contrario.

El "disparador" del proyecto fue la realización de un seminario en tres localidades diferentes (Chihuahua, Hermosillo y Monterrey) sobre la relación entre las CPAs y la planeación estratégica, donde participaron todos los maestros y directivos de la escuela. El seminario fue un ejercicio participativo de planeación para identificar 2, 3 o 4 programas estratégicos que fueran del interés de la comunidad escolar. Se pidió a los miembros de la escuela que seleccionaran

El efecto de la estructura de la escuela primaria en el funcionamiento de Comunidades... 
de manera completamente voluntaria el programa que más les interesara, y que estos grupos formados por interés trabajarían como CPAs. Se harían los arreglos para que en su horario de trabajo tuvieran por lo menos una hora a la semana para reunirse como CPA. Cada comunidad decidiría la agenda, los alcances de lo que querían hacer y las acciones que realizarían. Integrantes del equipo de investigación asistirían a las escuelas semanalmente para asesorar a las comunidades a solicitud de las mismas.

En Chihuahua participaron tres escuelas de características similares (número de maestros, alumnos y grupos). Las tres son primarias completas pertenecientes al subsistema federalizado, ubicadas en el área urbana. Sirven a comunidades con características socioeconómicas similares, y se encuentran en mismo sector de la ciudad. Una de ellas es una escuela de tiempo completo, con un horario de 8 a 3 . Las otras tienen un horario de 8 a 12:30. Este trabajo se refiere a los resultados en estas tres escuelas. La investigación consistió en tres estudios de caso. Cada escuela se consideró como un caso independiente. En cada escuela participó un asistente de investigación realizando observaciones en el sitio con visitas 4 días a la semana durante toda la jornada laboral entre enero y mayo del 2012. También se entrevistaron a las maestras y directoras de las 3 escuelas en el mes de mayo. Y se recolectaron documentos generados por maestros $\mathrm{y}$ directores durante el año escolar. La fuente principal de datos fueron las notas de campo, triangulándose con las entrevistas y los documentos recabados.

\section{Resultados}

Se conformaron 10 CPAs en las 3 escuelas de Chihuahua. Éstas se conformaron en octubre. Para ese momento del año escolar las escuelas ya tenían su plan de trabajo anual, y utilizaron la estructura típica de "comisiones" que caracteriza a las escuelas primarias. Cada maestro pertenecía a una "comisión" y responsable de alguna función de la escuela o algún proyecto del plan anual, normalmente incluido a petición de la autoridad. Aunque en el ejercicio de planeación participativa se identificaron una serie de necesidades que los maestros consideraban importantes para su escuela, al momento de que cada maestro seleccionó la necesidad más relevante para integrar las CPAs, la mayoría de los grupos se conformaron en función de la comisión a la que ya estaban asignados sus integrantes, y no en función de la necesidad que cada maestro, en lo individual, consideraba importante.

Sí me quedó claro que la idea era integrarse a una CPA de manera voluntaria, y con la CPA de la estrategia que uno consideraba más importante. Pero como ya estábamos en una comisión pensamos quedarnos los mismos, y trabajar con la comisión y con lo de la CPA. ¿Para qué estar en dos grupos? Luego iban a ser más reuniones, más actividades .... mejor con los mismos, aunque fuera algo distinto .... yo creo que eso evitó que avanzáramos, ya que a mí me interesaba más el proyecto de valores que el de los padres, que fue donde terminé (Maestra 2, Escuela C).

Nosotras avanzamos mucho, pero es porque ya habíamos trabajado juntas, y quedamos en la misma comisión este año. Así que cuando nos tocó la comunidad de los recreos pues ya estábamos juntas. Pero hubo otros, como las de matemáticas, que quedaron en esa comisión por sorteo ya que no decían en cuál querían estar. $\mathrm{Y}$ batallaron mucho porque no se conocían, y porque a unas ni las 
matemáticas ni lo de su comunidad les llaman la atención (Maestra 6, Escuela A),

Incluso en un caso las CPAs se integraron, con la idea de hacerlo "rápido", como estaban sentados en la mesa de trabajo sin ninguna consideración por el tema o los integrantes.

Quedamos en la misma comunidad porque llegamos temprano al seminario, y nos sentamos juntas porque nos llevamos bien. Pero los demás que llegaron tarde se sentaron donde había lugar, y cuando nos pidieron integrarnos a comunidades alguien dijo: "Así como estamos sentados". Así le hicieron el año pasado y el año anterior con las comisiones, porque hay gente con la que nadie se apunta. Y pues así quedamos. .... La CPA que no sabía para qué estaban juntos y que terminó deshaciéndose fue la de los que llegaron al final en [lugar de la sesión] (Maestra 4, Escuela B).

Este aspecto relacionado con la forma habitual de trabajo en la escuela tuvo un impacto importante en el funcionamiento de las CPAs. En dos casos facilitó el funcionamiento, cuando la "comisión" a la que pertenecían los maestros estaba relacionada directamente con la estrategia que se había incluido en la planeación. Este fue el caso de la CPA de "inclusión", formada por un grupo de maestros interesados en mejorar las prácticas y condiciones de la escuela para atender a niños con necesidades diferentes, fuera porque tenían niños con estas necesidades en su grupo, o porque anticipaban que tendrían niños con estas necesidades el ciclo escolar siguiente. Ésta era una situación que estaba presente desde hace 3 años en la escuela e iba en aumento.

Lo que pasa es que todos estamos preocupadas por los niños especiales. Y cada año llegan más. Pensamos que mejor trabajamos esto de una vez y no hasta que nos obliguen. 0 como dice [Maestra], "Mejor voy familiarizándome, porque me va a tocar [niña] y luego no voy a saber qué hacer". Pues sí. Si yo hubiera hecho eso el año pasado seguro hubiera batallado menos con [niño] de lo que batallé a principios del año (Maestra 1, Escuela C).

Pero de las 10 CPAs fue la excepción, al igual que otra CPA interesada en organizar los recreos para reducir los problemas y accidentes entre los niños por falta de espacio en la escuela. Esta estrategia se desarrolló en la escuela por indicación de la autoridad para hacer algo en respuesta al "bullying", una situación que se daba en la escuela sólo en tres casos muy identificados.

A mí siempre me ha interesado que los niños y las niñas tengan sus espacios, $\mathrm{y}$ que las niñas no sientan que los niños son los "dueños" de la escuela en el recreo. También que los grandes cuiden a los chicos, ya que corren y no se fijan y atropellan a los chiquitos. No es por mala intención. Es porque salen corriendo y no los ven. Y esto también le preocupa a [Maestra 6] y [Maestra 3]. El año pasado hablamos de hacer algo, y cuando nos preguntaron quién quería estar en la comisión de los recreos pues de inmediato nos apuntamos. Otra maestra nueva también quiso entrar a la comisión, y realmente hemos trabajado muy a gusto porque a ella también le interesa esto (Maestra 7, Escuela A).

La CPA estaba interesada en mejorar el ambiente en la escuela y que la distribución de espacios de recreación fuera más equitativo entre niños y niñas. En este caso las integrantes de la CPA aprovecharon la oportunidad de trabajar en este proyecto, independientemente de la razón formal de tener la comisión (i.e. la petición de la autoridad de hacer algo relacionado con el "bullying"). 
Las otras 8 CPAs no se conformaron realmente en función de su interés en la estrategia planteada. A pesar de la posibilidad de hacerlo, la mayoría de los maestros prefirió mantenerse con el grupo de "su comisión" y adherirse al proyecto que "les tocaba". Esta idea de "la comisión que me toca" se repitió en casi todas las entrevistas, incluso en las de las maestras de las dos CPAs que sí alinearon su comisión con la estrategia que iban a trabajar y su interés personal en tema, aprovechando el mandato de la autoridad. En consecuencia, el funcionamiento de las CPAs varió, aunque se dieron las condiciones organizacionales para que contaran con un espacio semanal para reunirse.

Identificamos 3 tipos diferentes de CPAs en términos de su funcionamiento a lo largo del año que podían anticiparse por la forma en la que la actividad de "integrar comisiones" impactó la integración de las CPAs,, independientemente de la directriz de integrarse de manera voluntaria en grupos con maestros basándose en intereses comunes.

1. CPAs orientadas al aprendizaje. Es el caso de las dos CPAs descritas (inclusión y recreos) que sí se conformaron basándose en intereses comunes. En estos dos casos (cada uno en una escuela diferente) los grupos funcionaron cómo está descrito en la literatura. Reflexionaron sobre lo que querían hacer y llegaron a acuerdos aceptados por todos. Se registró evidencia clara de crecimiento profesional y personal, y tomaron acciones con sus grupos y en sus clases que repercutieron en mejoras concretas a su práctica. Hacia el final del año ambas CPAs sabían qué actividades específicas incluir formalmente en el plan de trabajo anual.

2. CPAs orientadas a la acción. Este segundo tipo caracteriza a cinco de las
CPAs que se conformaron. En estos casos (presentes en las 3 escuelas) el grupo centraba sus discusiones, acuerdos $\mathrm{y}$ acciones a realizar programas diseñados por la CPA que respondían a necesidades planteadas de manera externa, ya fuera por la directora o por las autoridades educativas. Eran CPA ejecutoras. El hecho de poder reunirse y que tuvieran margen de decisión de lo que se iba a hacer llevó en por lo menos tres de los cinco casos a que se intentaran nuevas formas de enfrentar los problemas, como el caso de una comunidad que diseño, llevó a la práctica y ajustó una nueva forma de organizar a los padres de familia en la entrada y la salida de la escuela. 0 el caso de otra CPA que se organizó para que sus alumnos de $5^{0}$ y $6^{0}$ trabajaran juntos para que no se atrasaran los alumnos cuando las maestras tuvieran que faltar por una serie de cursos obligatorios ordenados por la autoridad. A pesar de que en un mes estas 3 maestras faltaron en días diferentes por atender cursos, sus alumnos tuvieron clases completas todos los días. En otros casos de su escuela y de otras los niños que no tenían maestra no iban a la escuela esos días. En mayor o menor medida estas cinco CPAs diseñaron, ejecutaron y evaluaron proyectos concretos de mejora relacionados con su práctica.

3. CPAs orientadas a la normatividad. En estos tres casos (uno por escuela) los grupos se reunían en el tiempo asignado, pero sólo para realizar lo que normativamente les correspondía. Ejecutaban lo que correspondía a su comisión, y el tiempo asignado para trabajo como CPA se utilizaba para darle seguimiento a su comisión, o en su defecto a realizar tareas individuales (revisión de trabajos, atender a padres de familia, etc.). En los tres casos hubo maestros que sistemáticamente no se reunían con su 
CPA. Y una de estas CPAs se deshizo de un día para otro, cuando tres de sus cinco integrantes abandonaron la escuela (dos jubilaciones $y$ un cambio). Los dos integrantes restantes se integraron a otra CPA de su escuela. Pero al preguntarles en qué estaban trabajando antes como CPA, ninguno de los dos pudo indicar explícitamente el propósito de esa CPA. "Creo que era algo académico. ¿Tal vez matemáticas" (Maestro 11, escuela B).

\section{Conclusiones}

Los hallazgos sugieren dos posibles conclusiones. En primer lugar la evidencia sugiere que prácticas administrativas como las CPA si pueden funcionar permitiendo que los maestros logran mejoras para toda la escuela que a la vez les permita aprender sobre temas de su interés y mejorar su práctica en el aula. Y que incluso pueden llevar a que los maestros logren mejoras en la escuela aunque estas mejoras no vayan acompañadas de mejoras en la práctica en el aula o a aprendizaje para resolver problemas en el futuro. En 7 de los 10 casos se lograron mejoras tangibles para las escuelas, El caso de las tres CPAs orientadas a la normatividad puede deberse a que algunos maestros trabajan en grupo de manera meramente nominal, una situación común en organizaciones educativas y en otras similares donde los profesionales tienen altos niveles de autonomía y donde la responsabilidad por el desempeño es individual (Mintzberg, 1997).

En segundo lugar la evidencia indica que las formas de organización tradicionalmente utilizadas en escuelas (la organización por "comisiones" en este caso) para asegurar el cumplimiento de demandas externas por medio de la participación de los maestros (lo que Hargreaves (1996) ha identificado claramente como "colonización administrativa") actúan en contra de la integración de CPAs y reducen considerablemente la posibilidad de que los maestros aprendan y mejoren su práctica en el aula al mientras hacen mejoras para la escuela. La evidencia de este estudio sugiere que pedirle a los maestros que contribuyan a mejorar la escuela dándoles más espacio para decidir qué problema tratar de resolver y cómo hacerlo, de preferencia en el proceso de elaboración del plan anual, puede ayudar a potenciar los resultados que la literatura sostiene pueden obtenerse con la práctica de CPAs. Valdría la pena hacer estudios en un futuro próximo asegurando realmente que las CPAs se integren en términos del interés por los maestros en la problemática específica a resolver y no en función de la práctica tradicional de las "comisiones" que se asocia a los planes anuales de trabajo.

\section{Referencias}

DuFour, R., Eaker, R., \& DuFour, R. (Eds.). (2005). On Common Ground: The Power of Professional Learning Communities. Bloomington, IN, EUA: Solution Tree.

Flores Kastanis, E., Flores Fahara, M., García Quintanilla, M., Rodríguez Bulnes, G., Holguín Ruiz, L. T., Olivas Maldonado, M., et al. (2007). Las escuelas públicas como Comunidades de Aprendizaje. In C. E. E. Bonilla (Ed.), Reseñas de Investigación en Educación Básica, Convocatoria 2003 (pp. 26). México, D.F.: Dirección General de Desarrollo de la Gestión e Innovación Educativa de la Subsecretaría de Educación Básica.

Flores Kastanis, E., Flores Fahara, M., García Quintanilla, M., Rodríguez Bulnes, G., Holguín Ruiz, L. T., Olivas

El efecto de la estructura de la escuela primaria en el funcionamiento de Comunidades... 
Maldonado, M., et al. (2009). Las escuelas públicas como comunidades de aprendizaje: Segunda parte. In L. A. Durán (Ed.), Reseñas de Investigación en Educación Básica, Convocatoria 2004 (pp. 28). México, D.F.: Secretaría de Educación Pública.

Flores-Kastanis, E. (2010). El maestro como trabajador de conocimiento y la promesa de una nueva escuela pública. In M. Flores Fahara \& M. Torres Herrera (Eds.), La escuela como organización de conocimiento (pp. 23-35). México, D.F.: Trillas.

Hargreaves, A. (1996). Profesorado, cultura y postmodernidad: Cambian los tiempos, cambia el profesorado (P. Manzano, Trans.). Madrid: Morata.

Lieberman, A., \& Miller, L. (Eds.). (2008). Teachers in Professional Communities: Improving Teaching and Learning. Nueva York: Teachers College Columbia University.

McLaughlin, M. W., \& Talbert, J. E. (2001). Professional Communities and the Work of High School Teaching. Chicago: The University of Chicago Press.

McLaughlin, M. W., \& Talbert, J. E. (2006). Building School-Based Teacher Learning Communities: Professional Strategies to Improve Student Achievement. Nueva York: Teachers College Press.

Mintzberg, H. (1997). La Organización Profesional (Editorial, Trans.). In H. Mintzberg, J. B. Quinn \& J. Voyer (Eds.), El Proceso Estratégico: Conceptos, Contextos y Casos (Breve ed., pp. 308-320). México, D.F.:

Prentice-Hall
Hispanoamericana.

Noffke, S. E., \& Somekh, B. (Eds.). (2009). The Sage Handbook of Educational Action Research. Londres: SAGE.

Reason, P., \& Bradbury, H. (Eds.). (2008). The Sage Handbook of Action Research: Participative Inquiry and Practice (2a. ed.). Londres: SAGE.

Senge, P. M. (2006). The Fifth Discipline: The Art and Practice of the Learning Organization (Revised ed.). Nueva York: Doubleday / Currency.

Whitford, B. L., \& Wood, D. R. (Eds.). (2010). Teachers Learning in Community: Realities and Possibilities. Albany, NY, EUA: State University of New York Press.

\section{Agradecimientos}

Esta investigación fue financiada por el Fondo Sectorial SEP/SEB-CONACYT (Convocatoria 2009). Agradezco al CONACYT, a la Subsecretaría de Educación Básica de la SEP, y a las autoridades educativas del estado de Chihuahua por su apoyo. Sobre todo agradezco a los maestros y directores de las 3 escuelas que participaron en el proyecto para mejorar el aprendizaje de sus alumnos. Fue un privilegio acompañarlos durante la mayor parte de un año y ver lo mucho que hacen por sus alumnos. 